\title{
Wenn ein Banker seine Weltsicht offenbart ... Ein offener Brief an Herrn Generaldirektor Mag. Andreas Treichl'
}

Am Freitag, den 28. Oktober 2016, wurde am Erste Bank Campus in Wien ein neues FinanzbildungsWorkshopzentrum, genannt FLIP („Erste Financial Life Park "), eröffnet. ${ }^{2}$ Nach Angaben des Vorstandsvorsitzenden der Erste-Group, Andreas Treichl, soll diese Einrichtung Kindern und Jugendlichen Finanzwissen für ihr eigenes Leben näherbringen. Dies ist zweifellos ein Anlass für die Vertreterinnen und Vertreter des Unterrichtsfaches Geographie und Wirtschaftskunde (GW) an Schulen, Pädagogischen Hochschulen und Universitäten, diese Initiative im Bereich von „Financial Literacy" zu begrüßen.

Die Finanzwirtschaft engagiert sich in Österreich seit mehr als 50 Jahren in diesem Bildungsbereich und unterstützt den schulischen GW-Unterricht bei der Erfüllung des gesetzlich vorgegebenen Auftrags zur wirtschaftlichen Erziehung mit Materialien und Fortbildungsseminaren. Zusätzlich werden Angebote von Seiten des Ministeriums für Soziales, Arbeit und Konsumentenschutz, der Bundeswirtschaftskammer, der Arbeiterkammer, des ÖGB, des Österreichischen Gesellschafts- und Wirtschaftsmuseums und weiterer Institutionen in Form von Lernmaterialien, Ausstellungen und Workshops in die Lehrer/innenaus- und -fortbildung sowie den Schulunterricht integriert.

\footnotetext{
Die Grundlage für diesen offenen Brief bildete ein „Basistext“, der auch für Stellungnahmen und Leserbriefe in den Tageszeitungen „Die Presse“ und „Kleine Zeitung“ diente, weswegen es in allen drei angeführten Quellen teilweise zu sinngemäß und auch wörtlich identen Formulierungen kommt, die hier nicht extra angegeben sind. Ein Hauptziel war und ist, eine möglichst rasche und weite Verbreitung dieser Stellungnahmen zu gewährleisten. Dieser offene Brief stellt eine erweiterte Version dieses ursprünglich formulierten „Basistexts“ dar.

2 Die Presse (28.10.2016): Sprühregen statt Geldregen für den Finanzminister. Wien. http://diepresse.com/home/ wirtschaft/eco 1848/5109458/Spruhregen-statt-Geldregenfur-den-Finanzminister?_vl_backlink=/home/index.do (28.10.2016). Der Standard (28.10.2016): Panne bei Festakt: Kalte Dusche am Weltspartag. Wien. http://derstandard. at/2000046632279/Kalte-Dusche-am-Weltspartag? ref=rec (28.10.2016). Kleine Zeitung (28.10.2016): Sprinkleranlage ausgelöst: Kalte Dusche für Finanzminister. Graz. http://www. kleinezeitung.at/wirtschaft/5109423/Weltspartag-bei-ErsteBank_Sprinkleranlage-ausgelöst_Kalte-Dusche (28.10.2016).
}

Dies erfolgt mit dem gemeinsamen Ziel, Schülerinnen und Schüler zu befähigen, mit Hilfe ihres durch die Finanzbildung gewonnenen ökonomischen Verständnisses als Konsumentinnen und Konsumenten sowie als künftige Erwerbstätige angemessene Entscheidungen zu treffen. Dabei geht es primär um eine Entfaltung der Orientierungs-, Urteils- und Handlungsfähigkeit von Heranwachsenden in einer zunehmend komplexer werdenden Gesellschaft.

Befremdlich neu ist für engagierte Schul- und Hochschullehrkräfte jedoch die Geringschätzung, die dem Fach "Geographie und Wirtschaftskunde" als Trägerfach der allgemeinbildenden sozioökonomischen Bildung in der Neuen Mittelschule und allgemeinbildenden höheren Schule durch Herrn Treichl bei diesem Anlass öffentlich entgegengebracht wurde. Nach dem unbeabsichtigten Duschen der geladenen Gäste durch eine defekte Sprinkleranlage fühlte sich Herr Treichl bemüßigt, gleich dem gesamten Schulsystem in Sachen Finanzbildung eine Kopfwäsche zu verpassen. Laut Online-Standard vom 28.10.2016 meinte er, dass ,in Sachen Finanzbildung die Kinder mit vollem Herzen dabei seien, die Lehrer leider nicht". Die Vermittlung dieser Inhalte an den Schulen sei „eine Katastrophe“, das Fach GW bezeichnete er als „Beleidigung“. Das wäre damit vergleichbar, wenn „Atomphysik und Leibesübungen in einem Fach unterrichtet" würden.

Schmerzt das? - Nur bedingt. Angelehnt an Bundeskanzler Christian Kerns Spruch „Ich entscheide immer noch selbst, wer mich beleidigt", stellt sich vielmehr eine gewisse Verwunderung ob des Gesagten ein. Warum begibt sich der Vorstandsvorsitzende einer Großbank auf so ein Niveau?

An der Zusammenarbeit von Schulen mit Partnern aus dem Finanzbereich kann es wohl nicht liegen. Seit Jahrzehnten gibt es einen regen Austausch zwischen Banken, Sparkassen und Schulen, finden Lehrausgänge in Banken statt, kommen Bankexpertinnen und -experten an Schulen. Wanderausstellungen informieren regelmäßig an verschiedenen Schulen über Wirtschaftsthemen, tausende Jugendliche haben im Rahmen des Schulunterrichts Kurse zum Erwerb des „Unternehmerführerscheins“ besucht. 
Lehrkräften dann zu unterstellen, „die Schüler seien interessiert, die Lehrer nicht", erscheint nicht nur weit hergeholt, sondern ist schlichtweg falsch. Herr Treichl sollte dies selbst am besten wissen, finden doch in „seinem“ FLIP allein im Herbst vier Lehrerfortbildungsveranstaltungen für sämtliche Schultypen $a b$ der 5. Schulstufe statt. Und wenn diese Workshops ein „Renner“ sind, dann sind sie es wegen der Lehrkräfte, die diese Workshops für ihre Schülerinnen und Schüler buchen und in ihren Unterricht einbauen. Desinteresse sieht anders aus.

Dass Herr Treichl offenbar kein Freund differenzierter Argumentation ist, lässt sich auch an anderen seiner Aussagen zeigen; so bezeichnete er im Mai 2011 Österreichs Politiker als „zu blöd und zu feig dazu und zu unverständig, weil sie von der Wirtschaft keine Ah-

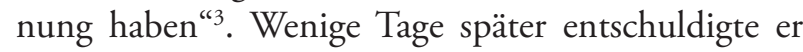
sich für die pauschale Entgleisung. Die Schlagzeile war es wohl wert.

Wenn ein für markige Sager bekannter Banker im Beisein des Finanzministers pauschale Anwürfe gegen alle Vertreterinnen und Vertreter eines Schulfaches macht, muss er sich jedoch einige entscheidende Fragen stellen lassen: Weiß Herr Treichl denn nicht, dass Themen der Wirtschafts- und Verbraucher/innenbildung prinzipiell multiperspektivisch und der politischen Bildung verpflichtet zu behandeln sind und somit thematische Anknüpfungspunkte zu einer Reihe von Bildungsanliegen haben? Das Unterrichtsfach GW, in dessen Zentrum der räumlich und wirtschaftlich handelnde Mensch steht, bietet dafür in den Lehrplänen viele Ankerpunkte in allen (!) Klassen der Sekundarstufen I und II. Es wurde daher auch vom Bildungsministerium als eines der drei Trägerfächer für Wirtschaftserziehung und Verbraucher/innenbildung genannt und nimmt diese Aufgabe auch wahr.

Vielleicht geht es Herrn Treichl wie zahlreichen Österreicherinnen und Österreichern, die in ihrer Schulzeit tatsächlich wenig bis nichts über Wirtschaft gehört haben. Da ist ein Schaden entstanden, keine Frage. Allerdings ist diese Zeit bereits einige Jahrzehnte vorbei. Deswegen die Arbeit engagierter Lehrkräfte zu diskreditieren, ist mehr als unfair und zeigt keinesfalls von guter Kenntnis der aktuellen Situation.

Es ist anzunehmen, dass Herr Treichl selbst tagtäglich wirtschaftliche Entscheidungen zu fällen hat, die eine Raumkomponente aufweisen, ob dies nun Standortfaktoren für die neue Zentrale der Erste Bank am Hauptbahnhof oder Fragen zur Filialstruktur im österreichischen Bankwesen sind. In Zeiten einer globalisierten Wirtschaft, in der jeder einzelne Mensch

Der Standard (14.5.2011): „Unsere Politiker sind zu blöd und zu feig“. Wien. http://derstandard.at/1304551930329/TreichlUnsere-Politiker-sind-zu-bloed-und-zu-feig (02.11.2016). bei seinen Konsumentscheidungen quasi in der ganzen Welt einkauft, klingt jedenfalls Herrn Treichls Vergleich, Geographie und Wirtschaftskunde sei wie „Atomphysik und Leibesübungen in einem Fach“, sehr realitätsfern.

In einem modernen GW-Unterricht steht der in gesellschaftlicher Bindung räumlich und wirtschaftlich handelnde Mensch im Mittelpunkt. Dieser lebt aber in einer zunehmend vernetzten Welt und daher kommt interdisziplinärem Denken eine immer größere Bedeutung zu. Gerade die Kategorie „Raum“ erfährt in Zeiten der Globalisierung eine immense Bedeutung, bedenkt man, wo Komponenten unserer Waren erzeugt werden, wohin Dienstleistungen ausgelagert werden, woher Menschen wandern, um ihr Streben nach wirtschaftlichem Glück zu verfolgen. GW ist in seinem interdisziplinären Ansatz geradezu ideal konzipiert, um Wissen über Wirtschaft, Mensch und Raum miteinander zu verknüpfen und für junge Menschen erlebbar zu machen.

Aufgrund welcher empirischen Befunde will Herr Treichl ableiten, dass GW-Lehrerinnen und -Lehrer bei der Vermittlung der Thematik versagen (in seiner Sprache „katastrophal“)? Laut Kleiner Zeitung will er es aus der Finanzkrise gelernt haben. Aber waren dafür nicht diejenigen verantwortlich, die nicht "nur“ eine allgemeine, sondern eine spezielle oder gar universitäre Ausbildung im Bereich Finanzwesen bzw. Wirtschaft vorweisen können? Dann würde hier nur einmal mehr die Verantwortung für gesellschaftliche Fehlentwicklungen an die Schulen weitergereicht. Oder sollte er damit auf fehlendes Finanzwissen verweisen, wie es in plakativ verkürzenden Befragungen fallweise konstatiert wird? Zum einen ist die ökonomische Aus- und Fortbildung der GW-Lehrer/innen an allen Standorten von Universitäten und Pädagogischen Hochschulen in Österreich auf einem hohen Niveau und vielfach in den letzten Jahrzehnten auch in den Curricula aufgewertet worden. Zum anderen muss man feststellen, dass die Kompetenz der Schülerinnen und Schüler in Bezug auf Finanzbildung nicht primär in der reproduktiven Gedächtnisleistung in für sie nur theoretisch vorstellbaren Begrifflichkeiten liegt (wie dies manche Studien insinuieren), sondern vielmehr darin eine wissensbasierte ökonomische Handlungskompetenz zu erlangen. Horst Knapp, nach dem auch ein begehrter Preis für Wirtschaftsjournalistinnen und -journalisten benannt ist, sprach vom Erwerb ökonomischer Intelligenz und nicht von nationalökonomischem Faktenwissen, wenn er die Schärfung ökonomischer Urteilskraft von der Schule forderte. ${ }^{4}$ Diese subjektorientierte lebensweltliche Zugangswei-

Knapp, H. (1979): Wider den ökonomischen Analphabetismus. In: Wissenschaftliche Nachrichten 51, 43-47. 
se war und ist auch oberstes Prinzip der sozioökonomischen Bildung innerhalb des Faches GW. Horst Knapp imponierte daher auch die Fachkenntnis der Aktienbesitzerinnen und -besitzer beim Interpretieren von Börsenkursen nicht besonders; wie wir nach acht Jahren Finanzkrise wissen, völlig zu Recht.

Wenn Fachkenntnisse einen so wichtigen Stellenwert bei ökonomischen Prozessen hätten, wären wohl auch die Ereignisse der letzten Jahre unerklärlich. Wir müssten daraus ein absolutes Versagen der klassischen wirtschaftlichen Ausbildung aller Wirtschaftsuniversitäten und vor allem der Entscheidungsträger/innen im Bankensektor ableiten. Denn auch die Erste Bank nahm eine damalige Liquiditätserhöhung aus Steuergeldern in der Höhe von hunderten Millionen Euro gerne an. Dies wäre ein vergleichbarer gedanklicher Kurzschluss, genauso wie den Vertreterinnen und Vertretern des Faches GW jegliche ökonomische Kompetenz abzusprechen.

Für eine erfolgreiche Finanzbildung der Schülerinnen und Schüler erscheint es zielführender, wenn sich schulische und außerschulische Bildung konstruktiv ergänzen und medial ausgerichtete Untergriffe unterbleiben. Es ist zu hoffen, dass Institutionen, Unternehmen und Interessenvertretungen der Wirtschaft weiterhin gemeinsam mit dem Fach GW das Ziel verfolgen, die (sozio)ökonomische Bildung junger Menschen zu unterstützen.

\section{Die Unterzeichner/innen:}

Mag. Felix Bergmeister, Fachdidaktik Geographie und wirtschaftliche Bildung, Institut für Geographie und Regionalforschung, Universität Wien

DP. in Carina Chreiska-Höbinger, BEd., Praxismittelschule der Pädagogischen Hochschule Wien; Fachgruppe für geographische und sozioökonomische Bildung der Österreichischen Geographischen Gesellschaft

Mag. ${ }^{a}$ Karin Dobler, Fachdidaktik Geographie und wirtschaftliche Bildung, Institut für Geographie und Regionalforschung, Universität Wien; Gutachterin für GWSchulbücher am Bundesministerium für Bildung

Prof. Mag. Dr. Johannes Dorfinger, Institut für digitale Kompetenz und Medienpädagogik, Pädagogische Hochschule Steiermark; Fachgruppe für geographische und sozioökonomische Bildung der Österreichischen Geographischen Gesellschaft

Prof. ${ }^{\text {in }}$ Mag. ${ }^{a}$ Elisabeth Dittrich, Bundesarbeitsgemeinschaft GWK an AHS; Fachgruppe für geographische und sozioökonomische Bildung der Österreichischen Geographischen Gesellschaft

Mag. Martin Dür, Fachbereich Geographie und Wirtschaftskunde, Pädagogische Hochschule Vorarlberg; Bundesarbeitsgemeinschaft GWK an AHS; Fachgruppe für geographische und sozioökonomische Bildung der Österreichischen Geographischen Gesellschaft.

Univ.-Prof. ${ }^{\text {in }}$ Dr. ${ }^{\text {in }}$ Heike Egner, RECC Geographie und Wirtschaftskunde, Alpen-Adria-Universität Klagenfurt

Mag. Johanna Eidenberger, Fachdidaktik Geographie und wirtschaftliche Bildung, Fachbereich sozialwissenschaftliche Bildung, Pädagogische Hochschule Oberösterreich

Prof. Mag. Wolfgang Fink, Bundesarbeitsgemeinschaft GWK an AHS, Landesfachkoordinator für Steiermark

Prof. Mag. Dr. Christian Fridrich, Fachbereich Geographie und Wirtschaftskunde, Pädagogische Hochschule Wien; Verein für Geographie und wirtschaftliche Bildung; Fachgruppe für geographische und sozioökonomische Bildung der Österreichischen Geographischen Gesellschaft

Mag. René Hanzlik, RGORG23 Anton Krieger Gasse; Fachdidaktik Geographie und wirtschaftliche Bildung, Institut für Geographie und Regionalforschung, Universität Wien

Prof. ${ }^{\text {in }}$ Silvia Hiebler, MAS, MSc; Institut für allgemeinbildende Fächer der Sekundarpädagogik, Fachbereich Geographie und Wirtschaftskunde, Pädagogische Hochschule Steiermark

Univ. Ass. ${ }^{\text {in }}$ Dr. ${ }^{\text {in }}$ Christiane Hintermann; Fachdidaktik Geographie und wirtschaftliche Bildung, Institut für Geographie und Regionalforschung, Universität Wien; Fachgruppe für geographische und sozioökonomische Bildung der Österreichischen Geographischen Gesellschaft.

Mag. Hartwig Hitz, Bundesarbeitsgemeinschaft GWK an AHS; Pädagogische Hochschule Niederösterreich; Institut für Geographie und Regionalforschung, Universität Wien; Fachgruppe für geographische und sozioökonomische Bildung der Österreichischen Geographischen Gesellschaft

Prof. Mag. Dr. Paul Hofmann, Fachbereich Geographie und Wirtschaftskunde, Pädagogische Hochschule Tirol; Bundesarbeitsgemeinschaft GWK an AHS; Institut für Geographie, Universität Innsbruck; Fachgruppe für geographische und sozioökonomische Bildung der Österreichischen Geographischen Gesellschaft

Prof. ${ }^{\text {in }}$ Dr. ${ }^{\text {in }}$ Maria Hofmann-Schneller, Fachgruppe für geographische und sozioökonomische Bildung der Österreichischen Geographischen Gesellschaft; Fachdidaktik Geographie und wirtschaftliche Bildung, Institut für Geographie und Regionalforschung, Universität Wien

Ass.-Prof. Mag. Dr. Thomas Jekel, Didaktik Geographie und Wirtschaftskunde, Universität Salzburg; Verein für Geographie und wirtschaftliche Bildung; Fachgruppe für geographische und sozioökonomische Bildung der Österreichischen Geographischen Gesellschaft

Ass.-Prof. Dr. Lars Keller, Institut für Geographie, Universität Innsbruck; Verein für Geographie und wirtschaftliche Bildung

Prof. Mag. Alfons Koller, Fachbereich Geographie und Wirtschaftskunde, Pädagogische Hochschule der Diö- 
zese Linz; Fachgruppe für geographische und sozioökonomische Bildung der Österreichischen Geographischen Gesellschaft; Verein für Geographie und wirtschaftliche Bildung

Mag. Wolfgang Kuschnigg, Fachbereich Geographie und Wirtschaftskunde, Institut für Ausbildung, Pädagogische Hochschule der Diözese Linz

Mag. Stefan Lamprechter, Fachbereich Geographie und Wirtschaftskunde, Kirchliche Pädagogische Hochschule Wien; Fachdidaktik Geographie und wirtschaftliche Bildung, Institut für Geographie und Regionalforschung, Universität Wien

Ao. Univ-Prof. Mag. Dr. Gerhard Karl Lieb, Institut für Geographie und Raumforschung, Universität Graz; Regionales Fachdidaktikzentrum Geographie und Wirtschaftskunde Graz; Verein für Geographie und wirtschaftliche Bildung; Fachgruppe für geographische und sozioökonomische Bildung der Österreichischen Geographischen Gesellschaft

Mag. ${ }^{a}$ Michaela Lindner-Fally, BORG Nonntal/Oberndorf; Fachdidaktik Geographie und wirtschaftliche Bildung, Fachbereich Geographie und Geologie, Universität Salzburg

Mag. ${ }^{a}$ Anna Oberrauch, Institut für Geographie, Universität Innsbruck; Fachgruppe für geographische und sozioökonomische Bildung der Österreichischen Geographischen Gesellschaft; Verein für Geographie und wirtschaftliche Bildung

Mag. Herbert Pichler, Fachdidaktik Geographie und wirtschaftliche Bildung, Institut für Geographie und Regionalforschung, Universität Wien; Verein für Geographie und wirtschaftliche Bildung

HS-Prof. ${ }^{\text {in }}$ Mag. ${ }^{a}$ Dr. ${ }^{\text {in }}$ Marlies Pietsch, Institut für allgemeinbildende Fächer der Sekundarpädagogik, Fachbereich Geographie und Wirtschaftskunde, Pädagogische Hochschule Steiermark

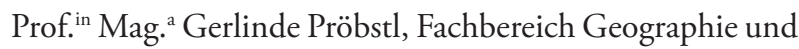
Wirtschaftskunde, Kirchliche Pädagogische Hochschule Wien; Fachdidaktik Geographie und wirtschaftliche Bildung, Institut für Geographie und Regionalforschung, Universität Wien

Mag. Peter Rak, Fachdidaktik Geographie und wirtschaftliche Bildung, Institut für Geographie und Regionalforschung, Universität Wien

Dr. Christian Reiner, Wirtschaftsforscher, Senior Researcher an der Lauder Business School, Wien.

Prof. Mag. Walter Scheidl, MSc.; Bundeslehrer im Hochschuldienst; GRG XXI, Franklinstraße 21; Institut für Bildungswissenschaft, Universität Wien; Institut für Geographie und Regionalforschung, Universität Wien

Prof. ${ }^{\text {in }}$ Mag. ${ }^{a}$ Doris Schneeweiß, Bundesarbeitsgemeinschaft GWK an AHS; Fachgruppe für geographische und sozioökonomische Bildung der Österreichischen Geographischen Gesellschaft

Mag. ${ }^{a}$ Dr. ${ }^{\text {in }}$ Ingrid Schwarz, Fachbereich Geographie und Wirtschaftskunde, Kirchliche Pädagogische Hochschule Wien; Fachdidaktik Geographie und wirtschaftliche Bildung, Institut für Geographie und Regionalforschung, Universität Wien; Fachgruppe für geographische und sozioökonomische Bildung der Österreichischen Geographischen Gesellschaft

Prof. Mag. Dr. Christian Sitte, Fachbereich Geographie und Wirtschaftskunde, Pädagogische Hochschule Niederösterreich; Fachgruppe für geographische und sozioökonomische Bildung der Österreichischen Geographischen Gesellschaft

Mag. Dr. Roman Stani-Fertl, Fachdidaktik Geographie und wirtschaftliche Bildung, Institut für Geographie und Regionalforschung, Universität Wien

Mag. ${ }^{a}$ Elisabeth Steininger, Institut für Fort- und Weiterbildung, Pädagogische Hochschule der Diözese Linz

Mag. ${ }^{a}$ Sandra Stieger, Fachdidaktik Ökonomie und Wirtschaftsgeographie, Fachbereich Geographie und Geologie, Universität Salzburg

Prof. ${ }^{\text {in }}$ Bbg Dr. ${ }^{\text {in }}$ Anke Uhlenwinkel, RECC Geographie und Wirtschaftskunde, Alpen-Adria-Universität Klagenfurt

Ao. Univ.-Prof. Dr. Christian Vielhaber, Fachdidaktik Geographie und wirtschaftliche Bildung, Institut für Geographie und Regionalforschung, Universität Wien

Robert Vogler, Senior Scientist, Fachbereich Geographie \& PLUS School of Education, Paris-Lodron-Universität Salzburg

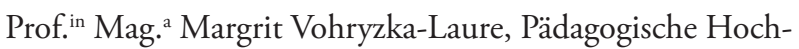
schule Kärnten, Bundesarbeitsgemeinschaft GWK an AHS; Fachgruppe für geographische und sozioökonomische Bildung der Österreichischen Geographischen Gesellschaft

Prof. Mag. Helmut Wagner, MSc, BHAK Krems; Fachgruppe für geographische und sozioökonomische Bildung der Österreichischen Geographischen Gesellschaft

Mag. Martin Welte, Bundesarbeitsgemeinschaft GWK an AHS; Fachgruppe für geographische und sozioökonomische Bildung der Österreichischen Geographischen Gesellschaft

Univ.-Prof. Mag. Dr. Helmut Wohlschlägl, Institut für Geographie und Regionalforschung, Universität Wien; Präsident der Österreichischen Geographischen Gesellschaft; Fachgruppe für geographische und sozioökonomische Bildung der Österreichischen Geographischen Gesellschaft 